\title{
Können wir uns dazu entscheiden, etwas zu glauben?
}

Abstract: In this essay I argue that, in a restricted sense, we can decide to believe certain propositions. It is conceded that acquiring a belief is not a basic action and possibly not even an action at all. However, this does not entail the impossibility of decisions to believe since not everything we can decide to do is a basic action. In fact, we can often decide to be in a certain state of affairs. Although beliefs normally aim at truth, there are cases in which we can voluntarily seek or avoid irrational states of affairs and thereby influence our attitudes. Bearing this in mind, situations in which we can talk of decisions to belief can be construed easily.

Keywords: doxastic voluntarism - beliefs - decisions - actions

\section{1}

Die Vorstellung, dass wir uns unabhängig von ihrer Wahrheit dazu entscheiden, eine bestimmte Proposition zu glauben, ruft bei vielen Missbehagen hervor. Während einige lediglich die moralische Verwerflichkeit solcher Entscheidungen anprangern (vgl. Clifford 1877), bestreiten andere sogar ihre Möglichkeit; diese werde ich im Rahmen dieses Essays diskutieren. Dabei bedarf jedoch die titelgebende Frage zunächst der Präzisierung. Erstens bezieht sich nämlich der Begriff „können“ offensichtlich immer auf eine Form von Möglichkeit. Derer gibt es jedoch bekanntlich viele und zwar unterschiedlich starke. Wir können von logischer und begrifflicher, von physischer und metaphysischer oder sogar von juristischer Möglichkeit sprechen und dies in allen Fällen in Form von „kann“-Sätzen tun. Zweitens ist es ebenso unklar, was genau der Begriff „wir“ bezeichnen soll. Bei abstrakten Überlegungen können damit vielleicht allgemein wirkliche oder mögliche Personen, rationale Wesen oder Handelnde gemeint sein, im Konkreten hingegen wird sich dieses „wir“ mangels Bekanntheit anderer intelligenter Lebensformen in der Regel auf Menschen beziehen. Aber geht es dabei um alle Menschen? Oder nur um einige? Oder gar um normale Menschen - und wenn ja: Was soll das sein? ${ }^{1}$

In diesem Essay werde ich zwei unterschiedliche Interpretationen der eingangs gestellten Frage besprechen, die ich sowohl für genuin interessant als auch für andere Bereiche (insbesondere den der ethics of belief, in dem die Frage bisher am ausführlichsten diskutiert wurde) am relevantesten halte:

1. Ist es überhaupt denkbar, dass sich irgendjemand dazu entscheidet, etwas zu glauben?

2. Haben Menschen faktisch die Fähigkeit, sich dazu zu entscheiden, etwas zu glauben?

\footnotetext{
${ }^{1}$ Danto und Morgenbesser etwa scheuen sich nicht, einen solchen Normalitätsbegriff (für Personen) einzuführen: „There is a basic repertoire $R$ of actions such that $a$ is a normal person only if $a$ possesses $R$.” (Danto \& Morgenbesser 1963, 436).
} 
Ich werde argumentieren, dass in beiden Fällen prima facie nichts dagegen spricht und dass die populärsten von „Doxastischen Involuntaristen“2 vorgebrachten Argumente die Möglichkeit solcher Entscheidungen nicht überzeugend zu widerlegen vermögen. Umgekehrt sind die von ihnen in Erwägung gezogenen Beispiele nicht so „exotisch“, wie bisweilen suggeriert wird.

\section{2}

Zunächst soll also geklärt werden, ob es überhaupt denkbar ist, dass wir uns dazu entscheiden können, etwas zu glauben. Da sich dies nicht nur sprachlich problemlos ausdrücken lässt, sondern ich auch unterstelle, dass wir zumindest eine sehr vage Vorstellung davon haben, was es bedeuten könnte, sehe ich dabei die Beweislast aufseiten des Involuntaristen. Ich werde zunächst zwei involuntaristische Einwände besprechen, die das Verhältnis der Begriffe „glauben“ und „entscheiden“ betreffen. Ich kann in diesem Rahmen keine erschöpfenden Definitionen anbieten, ${ }^{3}$ sondern werde vielmehr besagte Einwände daraufhin prüfen, ob sie angesichts unserer Verwendung dieser Begriffe in anderen Kontexten haltbar sind. Dies wird uns möglicherweise auch bereits dabei helfen, die Umstände näher einzugrenzen, unter denen solche Entscheidungen möglich sein könnten.

Beginnen wir mit der empirisch weitestgehend unkontroversen Feststellung, ${ }^{4}$ dass wir nicht „einfach so“"etwas glauben können. Diese scheint von einigen Autoren bereits damit gleichgesetzt zu werden, dass wir uns nicht dazu entscheiden können, etwas zu glauben. Williams etwa erklärt in seinem klassisch gewordenen Aufsatz "Deciding to Believe" zunächst: "The ultimate focus of my remarks is going to be on the relations between belief and decision and certain puzzles that arise about the relation between these two ideas." (Williams 1973, 136) Mit seinem Hauptargument, zu dem ich noch kommen werde, bestreitet er jedoch lediglich die Möglichkeit, nach Belieben zu glauben (believe at will), ohne zu erläutern, ob und wenn ja inwiefern sich diese beiden Formulierungen unterscheiden. Nach Belieben glauben zu können scheint jedoch zu bedeuten, dass ein Glaube unmittelbar durch eine entsprechende Intention, eine beliebige Proposition p zu glauben, erworben werden kann. Entsprechend behauptet Winters: "[T]he philosophical controversy about belief at will concerns whether the model of free basic action can be applied to belief acquisition." (Winters 1979, 244)

\footnotetext{
${ }^{2}$ Gemäß einer gängigen (wenngleich fragwürdigen, weil ihre erläuterte Ambiguität unterschlagenden) Klassifikation werden diejenigen, die die eingangs gestellte Frage bejahen, als „Doxastische Voluntaristen“ und diejenigen, die sie verneinen, als Doxastische „In-“ oder „Anti-Voluntaristen“ bezeichnet.

${ }^{3}$ Einen Ausgangspunkt für eine Definition von Entscheidungen könnte McCann bilden. Dieser definiert sie als das Auftauchen von Intentionen. (McCann 1998, 135ff.) Allerdings berücksichtigt er dabei nicht die Möglichkeit, sowohl entsprechend dieser Intentionen zu handeln als auch nicht zu handeln, die mir eine notwendige Bedingung für Entscheidungen zu sein scheint.

${ }^{4} \mathrm{Zu}$ einer Gegenposition siehe Ryan 2003.
} 
Zugegeben, wenn es irgendetwas gibt, zu dem wir uns entscheiden können, dann sind das Basishandlungen, also solche Handlungen, die wir direkt ausführen können, ohne dafür etwas anderes tun zu müssen (vgl. Danto 1965). Wir können jedoch auch sinnvoll davon sprechen, dass sich jemand entscheidet Squash zu spielen, ein Haus zu bauen oder Arzt zu werden. Solche komplexen Handlungen können sich über lange Zeiträume erstrecken und sich aus etlichen Teilhandlungen zusammensetzen. Ihrer Komplexität scheinen lediglich insofern Grenzen gesetzt zu sein, als ihr Gelingen zu einem bestimmten Maß durch unsere (basaleren) Handlungen beeinflussbar sein muss. Die Behauptung, etwas zu glauben wäre eine Basishandlung beziehungsweise wir könnten es nach Belieben, ist also um einiges stärker als die, dass wir uns entscheiden können, etwas zu glauben, weshalb eine Widerlegung ersterer nicht notwendigerweise mit einer Widerlegung letzterer einhergehen muss.

Vielleicht liegt das Problem aber auch gar nicht darin, dass etwas zu glauben keine Basishandlung ist, sondern darin, dass es überhaupt keine Handlung ist? Überzeugungen werden üblicherweise als Zustände von Subjekten beziehungsweise als Relationen zwischen Subjekten und Propositionen verstanden (vgl. Alston 1988, 261f.). Etwas zu glauben würde demnach weniger bedeuten etwas zu tun als vielmehr etwas zu sein.

Auch damit ist aber nicht ausgeschlossen, dass man sich entscheiden kann etwas zu glauben. Zum einen ist es nämlich oft gar nicht so einfach, Handlungen und Zustände auseinanderzuhalten. Wenn ich beispielsweise eine Handlung $X$ ausführe, dann bin ich immer auch gleichzeitig jemand, der Xt (ein Xer). Der Zustand ist in solchen Fällen nicht etwa das Resultat einer Handlung, sondern lediglich eine andere Beschreibung derselben. Wenn ich mich aber entscheiden kann zu Xen, dann kann ich mich auch entscheiden ein Xer zu sein, solange sich meine Entscheidung auf die seinBeschreibung bezieht; ebenso wie Intentionen scheinen Entscheidungen nämlich intensional, also beschreibungsgebunden, zu sein. Wenn wir etwa von jemandem verlangen „Sei nett!“, dann unterstellen wir gerade, dass das Nettsein etwas ist, das jemand tun kann, indem er etwa lächelt oder Smalltalk hält.

Zum anderen - und diese Fälle werden für uns relevant sein - können wir uns oft dazu entscheiden, Handlungen auszuführen, die Zustände verursachen. Der Satz „S trägt einen Hut“ etwa scheint prima facie einen Zustand zu beschreiben und keine Handlung. Selbst Schlafende oder gar Schaufensterpuppen, die klarerweise keine Agenten sind, können Hüte tragen; wer einen Hut trägt, tut damit nichts im engeren Sinne. Dennoch würde niemand behaupten, dass wir uns nicht entscheiden können Hüte zu tragen. Es könnte zum Beispiel sein, dass sich S entschieden hat, den Hut aufzusetzen. Vielleicht hat sie sich aber auch entschieden, jemand anderen darum zu bitten, ihr den Hut aufzusetzen. Oder er wurde ihr ursprünglich gegen ihren Willen aufgesetzt, 
aber sie hat sich dazu entschieden, ihn aufzulassen, als sie die Möglichkeit gehabt hätte ihn abzusetzen. In allen drei Fällen können wir offenbar sinnvoll behaupten, dass sich $\mathrm{S}$ dazu entschieden hat, den Hut zu tragen.

Wie genau Handlungen beziehungsweise Unterlassungen auf diese Weise mit Zuständen zusammenhängen können und ob letztere in solchen Fällen nicht doch als (komplexe) Handlungen zählen sollten, vermag ich hier nicht zu sagen. Vielleicht muss aber auch nicht alles, zu dem wir uns entscheiden können, eine Handlung sein. Zumindest scheint mir offensichtlich, dass die Verursachung oder Aufrechterhaltung von Zuständen nicht damit identisch ist, sich in ihnen zu befinden, dass wir uns aber dennoch in vielen Fällen zu letzterem entscheiden können. Voraussetzung dafür scheint neben der Möglichkeit diesen Zustand hervorzurufen oder aufrechtzuerhalten lediglich $\mathrm{zu}$ sein, dass sich die korrespondierende Intention auf das Handlungsergebnis beziehen kann, was im Falle von Glauben fraglos möglich ist. ${ }^{5}$

\section{3}

Auf begrifflicher Ebene spricht also nichts dagegen, sich zu einem Glauben zu entscheiden. Könnte es aber vielleicht aus anderen Gründen undenkbar sein? Trotz der bemängelten Ungenauigkeiten möchte ich hierzu noch auf ein Argument von Williams (Williams 1973) eingehen, der ebendies zu zeigen versucht. Williams stellt zunächst fest, dass es eine zentrale Eigenschaft von Überzeugungen ist, auf Wahrheit abzuzielen (aim at truth): Etwas zu glauben bedeutet, es für wahr zu halten und in der Regel Gründe dafür angeben zu können. Das Problem, das er bezüglich willentlich erworbener Überzeugungen aufwirft, lässt sich nun folgendermaßen zuspitzen: Wie könnte ich etwas für wahr halten, wenn ich gleichzeitig wüsste, dass ich diese Einstellung willentlich und damit nicht allein abhängig von ihrer Wahrheit erworben habe? Selbst wenn ich im Nachhinein Gründe für diesen Glauben angeben könnte, so wüsste ich doch, dass es nicht diese Gründe waren, die mich den Glauben annehmen ließen; möglicherweise hatte ich vorher sogar bessere Gründe dafür, ihn abzulehnen. Williams sieht dies als Indiz dafür an, dass Glauben nach Belieben (siehe oben) nicht nur kontingenterweise unmöglich ist, sondern dass bereits seine Denkbarkeit fraglich ist.

Schon Winters (1979) kritisiert dieses Argument und unterscheidet zunächst die Tatsache, dass ein Glaube willentlich erworben wurde, von dem Glauben daran, dass er willentlich erworben

\footnotetext{
5 Hieronymi scheint mir über ihr Ziel, Überzeugungen als Nicht-Handlungen zu entlarven, hinauszuschießen, wenn sie behauptet, dass bereits die Intention, etwas zu glauben, eigentlich (properly speaking) als Intention, diesen Glauben zu verursachen, verstanden werden müsste (Hieronymi 2008). Insbesondere mutet es seltsam an von Intentionen, die wie erwähnt intensional sind, zu behaupten, sie würden sich „eigentlich“ auf etwas anderes beziehen.
} 
wurde. Sie weist darauf hin, dass nur letzterer mit diesem neuen Glauben inkompatibel ist, nicht aber erstere. Darauf aufbauend ersinnt Bennett (1990) mit den „Credamiten“ eine fiktive Gemeinschaft, deren Mitglieder die Fähigkeit haben, sich selbst nach Belieben Überzeugungen zu induzieren. Außerdem - und das ist das Entscheidende - vergessen sie es jedes Mal, wenn sie von dieser Möglichkeit Gebrauch machen. Werden Credamiten mit Beweisen dafür konfrontiert, dass eine ihrer Überzeugungen auf diese Weise zustande kam, müssen sie diese entweder ignorieren oder aber den neuen Glauben ablegen - es sei denn, sie haben inzwischen neue Evidenzen entdeckt, die ihn unabhängig von ihrer Entscheidung begründen. Bennett schließt also gerade den inneren Konflikt aus, den Williams für so problematisch hält: "So each Credamite knows that he sometimes wills himself to believe something, even though it is never true that he now has a belief which he now remembers having willed himself to acquire." (Bennett 1990, 93)

Obwohl Williams`Argument damit grundsätzlich widerlegt ist, scheint mir sein Gedankengang in abgewandelter Form bedenkenswert. Spinnen wir Bennetts Gedankenexperiment einmal dahingehend weiter, dass Credamiten jede Überzeugung, derer sie sich bewusst werden, früher oder später willentlich ins Gegenteil verkehren würden und ferner wüssten, dass alle Mitglieder ihrer Spezies dies so handhaben. Unter diesen Umständen wüssten sie von allen ihren Überzeugungen, dass diese, sofern sie ihnen schon einmal bewusst waren, mindestens einmal willentlich beeinflusst wurden. Damit müssten sie aber bei allen ihren Überzeugungen davon ausgehen, dass sie diese mit ernstzunehmender Wahrscheinlichkeit nicht wegen der Gründe, die ihnen dafür einfallen, erworben haben, sondern durch einen Willensakt. Unter diesen Bedingungen würde es tatsächlich schwierig für sie, irgendetwas zu glauben und für begründet zu halten. ${ }^{6}$ Dieses Problem verschwindet nur schleichend, wenn wir den Anteil der Überzeugungen, auf die Credamiten ihre Fähigkeit anwenden, allmählich senken. Diese Überlegungen scheinen mir nahezulegen, dass selbst Credamiten, um weiterhin Überzeugungen haben zu können, davon ausgehen müssen, dass diese im Normalfall, der sich hier freilich nicht genau beziffern lässt, auf Gründen fußen und nicht auf Willensakten.

\section{4}

Können wir uns also dazu entscheiden, etwas zu glauben? Wenn wie gezeigt a priori nichts dagegen spricht, lässt sich diese Frage auf die konkreten menschlichen Fähigkeiten beziehen. Der beste Beweis dafür, dass Menschen eine solche Fähigkeit haben, wären freilich Beispiele dafür, dass sie sie bereits erfolgreich ausüben. Dabei wären schon Fälle interessant, in denen einzelne und vielleicht außergewöhnliche Menschen dies zu tun vermögen. Die Beispiele, die ich

\footnotetext{
${ }^{6}$ Schließlich müsste sich ihr Zweifel sogar gegen die Überzeugung richten, dass jede ihrer Überzeugungen potentiell unbegründet ist, womit sie dem klassischen Dilemma des radikalen Skeptikers ausgesetzt wären.
} 
vorstellen werde, sollten jedoch deutlich machen, dass es sich um eine Fähigkeit handelt, die mindestens die allermeisten Menschen besitzen.

Es wurde bereits dargelegt, dass etwas zu glauben sicherlich keine direkte und vielleicht überhaupt keine Handlung ist, sondern vielmehr ein Zustand, in dem man sich befindet. Auf der Suche nach Beispielen sollten wir uns also auf Handlungen oder Unterlassungen konzentrieren, mithilfe derer wir Überzeugungen verursachen oder aufrechterhalten können - ähnlich dem Aufsetzen oder Nicht-Absetzen eines Hutes. Nun ist es Konsens, dass wir Meinungen wenigstens manchmal kausal erklären können, sofern wir über ihre Genese sprechen und Ursachen für sie angeben - wenngleich für die Erforschung dieser Beziehungen traditionellerweise nicht die Philosophie zuständig ist, sondern vielmehr die Psychologie sowie die Sozialwissenschaften. Selbst Williams, der bezüglich der Möglichkeit von Glaubensentscheidungen skeptisch ist, erwägt, dass wir uns diese Kausalbeziehungen zur Beeinflussung unserer Überzeugungen zunutze machen könnten:

"[I]t may be said that there is room for the application of decision to belief by more roundabout routes. For we all know that there are causal factors, unconnected with truth, which can produce belief: hypnotism, drugs, all sort of things could bring it about that I believe that $p$. Suppose a man wanted to believe that $p$ and knew that if he went to a hypnotist or a man who gave him certain drugs he would end up believing that $p$. Why could he not use this roundabout method [...]?" (Williams 1973, 149)

Williams kommt zu dem Schluss, dass wir uns mittels solcher "roundabout methods" durchaus dazu entscheiden können, etwas zu glauben, sofern beim Entscheidenden "non-truth-centred motives" vorliegen, er also genuin daran interessiert ist, eine Proposition zu glauben, unabhängig davon, ob sie wahr ist. ${ }^{7}$

Alston, der regelmäßig als rigider Involuntarist zitiert wird, kommt zu dem Schluss, dass wir keinen ausreichenden Grad an Kontrolle haben, um eine Position zu begründen, die er "the deontological conception of the epistemic justification of belief" nennt, räumt jedoch die Möglichkeit indirekter Beeinflussungen der eigenen Überzeugungen ebenfalls ein:

"It does seem that we have some degree of long range voluntary control over at least some of our beliefs. As just noted, people do set out on long run projects to get them to believe a certain

\footnotetext{
${ }^{7}$ Tatsächlich scheint zusätzlich zur oben genannten Beschränkung die Zahl der Fälle, in denen Menschen so etwas tun, schon allein dadurch begrenzt, dass sie faktisch meistens ein starkes Interesse daran haben, dass ihre Überzeugungen der Wahrheit entsprechen.
} 
proposition, and sometimes they succeed in this. Devices employed include selective exposure to evidence, selective attention to supporting considerations, seeking the company of believers and avoiding non-believers, self-suggestion, and (possibly) more bizarre methods like hypnotism. By such methods people sometimes induce themselves to believe in God, in materialism, in Communism, in the proposition that they are loved by X, and so on." (Alston 1988, 275)

Die Bezeichnung "long range", wenngleich Alston damit lediglich meint, dass die Kontrolle nicht durch eine ununterbrochene intentionalene Handlung ausgeübt werden kann, suggeriert allerdings, solche Beeinflussungen würden stets einige Zeit in Anspruch nehmen müssen. Zudem unterschätzt Alston offenbar sowohl die häufig als auch die Erfolgsrate solcher Projekte deutlich (vgl. Alston 1988, 276).

Unter den Möglichkeiten, die Williams und Alston vorbringen, finden sich bereits einige, die im Alltag vielfältig exemplifiziert werden. So wird zwar in näherer Zukunft wohl keine Pille erfunden werden, die den Glauben daran induziert, dass die USA noch immer eine britische Kolonie sind (vgl. Alston 1988, 263), aber Williams“ Verweis auf Drogen ist dennoch nicht abwegig: Bereits geringe Mengen Alkohol etwa können Menschen glauben lassen, das Leben sei schön, ihre Sorgen seien gar nicht so wichtig, sie sähen blendend aus und vieles mehr. Oft genug wird Alkohol ja zu genau diesem Zweck konsumiert - von illegalen Drogen und ihren Effekten ganz zu schweigen.

Auch die von Alston erwähnte Autosuggestion ist keinesfalls „,bizarr“. Beispielsweise wissen viele Sportler oder Bewerber ihre Erfolgschancen dadurch zu steigern, dass sie effektive Techniken entwickeln, daran zu glauben, dass sie erfolgreich sein werden, ohne dass sie neue Evidenzen dafür sammeln würden. Zudem ist es eines der Axiome der kognitiven Verhaltenstherapie, einer der am weitesten verbreiteten und erfolgreichsten Psychotherapiemethoden, dass unsere Überzeugungen unsere Affekte beeinflussen und dass eine Regulation ersterer eine Regulation letzterer bewirken kann. ${ }^{8}$ Unabhängig davon, ob diese Theorie stimmt und wie irrational die Haltungen von Patienten manchmal sein mögen, werden sie sich, sofern sie der Methode vertrauen, häufig dazu entscheiden, mithilfe verschiedener Techniken Einfluss darauf zu nehmen, damit es ihnen besser geht.

Schließlich scheint es mir erwähnenswert, dass wir uns oft dazu entscheiden können, Situationen auszuweichen, von denen wir wissen, dass wir in ihnen dazu neigen, an Rationalität einzubüßen. Phobiker etwa werden bei Konfrontation mit entsprechenden Stimuli nicht nur ängstlich, sie

\footnotetext{
${ }^{8}$ Diese Darstellung ist insofern etwas verkürzt, als der Begriff der Kognition wesentlich mehr umfasst als Überzeugungen, die jedoch zweifellos einen Bestandteil ausmachen (vgl. Beck 1979). Siehe auch Whiting 2006 sowie Gipps 2013 zu philosophischen Betrachtungen dieser Schule.
} 
neigen auch dazu, daran zu glauben, dieser sei tatsächlich gefährlich, selbst wenn sie dies in neutralen Situationen nicht tun; ${ }^{9}$ sie können sich entsprechend entscheiden, an ihrem rationaleren Glauben festzuhalten, indem sie sich entscheiden, den Stimulus zu meiden. Ähnliches ist aber fraglos auch im nicht-pathologischen Bereich möglich: Eine Mutter, deren Tochter spätabends noch nicht von einer Feier zurück ist, wird sich hüten, sich einen Horrorfilm anzusehen, in dem feiernde Teenager bevorzugt entführt und grausam ermordet werden, denn dies könnte bewirken, dass sie sich unnötig Sorgen macht. Sie kann sich also dazu entscheiden, an ihrem rationaleren Glauben („Teenager werden nur äußerst selten entführt.“) festzuhalten, indem sie sich dazu entscheidet, Handlungen zu unterlassen, die diesen Glauben ins Wanken bringen würden.

Natürlich können und sollen diese Beispiele nicht beweisen, dass wir alle unsere Überzeugungen beliebig oder auch nur indirekt beeinflussen können; wie erläutert scheint mir auch ein grundsätzliches Vertrauen darauf, dass sie auf Wahrheit abzielen, gerade eine Bedingung dafür, punktuell davon abzuweichen. Ich hoffe jedoch gezeigt zu haben, dass ihre Beeinflussung nicht nur denkbar, sondern bereits Bestandteil einiger ganz alläglicher Praktiken ist. Wer die Beweiskraft dieser Beispiele bestreitet, muss, so scheint mir, mit zweierlei Maß messen und einen engeren Begriff von Entscheidungen anlegen, als wir dies in anderen Kontexten tun. Ebenso wie die Vorstellung, wir seien unseren Gefühlen willenlos ausgeliefert, vielen inzwischen als antiquiert und romantisierend gilt, sollten wir vielleicht allmählich Abstand nehmen von der Idee, unsere Meinungen seien etwas, das uns ganz und gar unabhängig von unserem Willen zukommt.

\section{Anmerkungen:}

Ich danke Prof. Dr. Oliver Hallich und den Teilnehmenden seines Oberseminars für die Möglichkeit, meine Argumentation ausführlich zu diskutieren. Ferner danke ich Prof. Dr. Thomas Grundmann, Sebastian Schmidt sowie Matthias Bau und Ilka Fladung für hilfreiche Anmerkungen.

\section{Literatur:}

Alston, William P. 1988. "The Deontologic Coneception of Epistemic Justification." Philosophical Perspectives 2, 257-299.

Beck, Aaron T. 1979. Wahrnehmung der Wirklichkeit und Neurose. Kognitive Psychotherapie emotionaler Störungen . München: Pfeiffer.

Bennett, Jonathan 1990. "Why is Belief Involuntary?” Analysis 50 (2), 87-107.

Clifford, William K. 1877. "The Ethics of Belief.” The Contemporary Review 29, 289-309.

\footnotetext{
${ }^{9}$ Vgl. Beck 1979, 136ff. sowie Pössel \& Holzhay 2006.
} 
Danto, Arthur C. \& Morgenbesser, Sidney 1963. "What we can do." The Journal of Philosophy 60 (15), 435-445.

Danto, Arthur C. 1965. “Basic Actions.” American Philosophical Quarterly 2 (2), 141-148.

Gipps, Richard G. T. 2013. “Cognitive Behavior Therapy. A Philosophical Appraisal.” In: The Oxford Handbook of Philosophy and Psychiatry, hrsg. von K. W. M. Fulford, Martin Davies \& Richard G. T. Gipps. Oxford: Oxford University Press, 1245-1263.

Hieronymi, Pamela 2008. “Responsibility for believing.” Synthese 161 (3), 357-373.

McCann, Hugh J. 1998. The Works of Agency. On Human Action, Will, and Freedom. Ithaca (NY): Cornell University Press.

Pössel, Patrick \& Holzhay, Anja 2006. "Test of the Dual-Belief System in Women with and without Phobic Fear of Spiders. A Pilot Study." Clinical Psychology and Psychotherapy 13 (4), 246-255.

Ryan, Sharon 2003. "Doxastic Compatibilism and the Ethics of Belief." Philosophical Studies $114(1 / 2), 47-79$.

Williams, Bernard 1973. "Deciding to Believe." In: Problems of the self. Philosophical papers 1956-1972, hrsg. von Bernard Williams. Cambridge: Cambridge University Press, 136-151.

Whiting, Demian 2006. "Why Treating Problems in Emotion May Not Require Altering Eliciting Cognitions.” Philosophy, Psychiatry, \& Psychology 13 (3), 237-246.

Winters, Barbara 1979. "Believing at Will.” The Journal of Philosophy 76 (5), 243-256. 